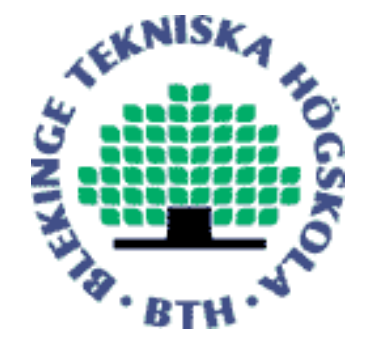

Copyright (C) 2011 IEEE.

Citation for the published paper:

A Mobility Management Solution for Simultaneous Mobility with mSCTP

Kaibo Zhou, Gang Liu, Yong Yao, Adrian Popescu

4th IFIP International Conference on New Technologies, Mobility and Security

2011 Paris

This material is posted here with permission of the IEEE. Such permission of the IEEE does not in any way imply IEEE endorsement of any of BTH's products or services Internal or personal use of this material is permitted. However, permission to reprint/republish this material for advertising or promotional purposes or for creating new collective works for resale or redistribution must be obtained from the IEEE by sending a blank email message to pubs-permissions@iee.org.

By choosing to view this document, you agree to all provisions of the copyright laws protecting it. 


\title{
A Mobility Management Solution for Simultaneous Mobility with mSCTP
}

\author{
Kaibo Zhou, Gang Liu \\ China Academy of Telecom Research (CATR), 100191 Beijing, China \\ Email: \{zhoukaibo, liugang\}@ catr.cn \\ Yong Yao, Adrian Popescu \\ School of Computing, Blekinge Institute of Technology (BTH), 37179 Karlskrona, Sweden \\ Email: \{Yong.Yao, Adrian.Popescu\}@bth.se
}

\begin{abstract}
The paper is about mobile Stream Control Transmission Protocol (mSCTP) and the problems related to simultaneous mobility. Simultaneous mobility is when the both endpoints of a communication session are mobile and they move at about the same time.

mSCTP works well in the case of non-simultaneous mobility where the SCTP association is established between a mobile endpoint and a stationary one. In the case of simultaneous mobility however, the probability of broken association may become high because both endpoints may suffer from losing address binding update. This is a consequence of the fact that the targeted addresses become unreachable.

In this paper, we suggest a solution based on Host Name Address (HNA), together with pro-active Name Server, Address Handling Function (AHF) and Simultaneous Mobility Detection Function (SMDF) to eliminate this problem. Our preliminary results show that the performance of our solution is as good as for non-simultaneous mobility situation in terms of packet loss rate for low rate stream. On the other hand, the drawback is that some modifications to the current standard MSCTP are needed.
\end{abstract}

Index Terms-Mobile SCTP, Simultaneous Mobility, Host Name Address, Name Server

\section{INTRODUCTION}

Stream Control Transmission Protocol (SCTP) was initially designed for the transmission of SS7 signaling across packet network. SCTP is a general-purpose transport protocol for message-oriented applications, which can be used in place of both TCP and UDP [1], [2]. The SCTP basic service is the reliable transfer of user messages (so-called streams) between peer SCTP users. SCTP performs this service within the context of an association between two SCTP endpoints. SCTP is connection-oriented in nature, but the SCTP association is broader than the TCP connection. This means that each SCTP endpoint provides the other endpoint (during association startup) with a list of transport addresses (i.e., multiple IP addresses in combination with a SCTP port) through which the endpoint can be reached and from which it can generate SCTP packets. The association works for transfers over all possible source/destination combinations that can be generated from each endpoint list. This means that an SCTP association is more resilient to network failures and provides multi-homing facilities.

mobile SCTP (mSCTP) is an extension of SCTP designed to solve the problem of vertical handover in heterogeneous networks by dynamically switching between alternate network interfaces [3]. The extension consists of the addition of Dynamic Address Reconfiguration (DAR) [3] feature, which is a mobility enabling feature but not a mobility solution by itself. DAR allows SCTP to dynamically add or delete IP addresses while keeping the e2e connection intact, i.e., during an active SCTP association.

However, a serious problem related to these protocols is because of the basic assumption that one mobile endpoint communicates with the corresponding stationary endpoint only. This assumption does not match the situations existent today in real mobile networks, where both endpoints can be mobile and we accordingly have the problem of simultaneous mobility. The endpoints can therefore simultaneously move and update address binding. This calls for new, smarter solutions for mobility, which are able to handle such situations.

There are several solutions suggested to solve this problem. For instance, in [4], the authors provide a very detailed analysis of the problem of simultaneous mobility. An analytical framework is introduced for analyzing certain mobility problems including but not limited to simultaneous mobility. Solutions are suggested to handle the simultaneous mobility problem for Mobile IPv6 (MIPv6), MIP with Location Register (MIP-LR) and SIP Mobility Management (SIPMM).

Another important work is [5], where the authors mention two solutions, namely SCTP with Mobile IP [6] and using dynamic DNS [7]. A solution, called mSCTP with Reliable Server Pooling (RSerPool), is illustrated in detail to cope with the simultaneous handover problem. It acts like a shim layer between L4 and L5 (Internet Model). An important drawback of the solution SCTP with Mobile IP (MIP) is that the endpoint must support MIP and potential large handover delay may be introduced because of the well-known MIPv4 problem of triangle routing. On the other hand, for the solution using dynamic DNS, the problem is that DNS has been designed under the assumption that its entries change infrequently and the connection is not reliable because it is based on UDP.

In this paper, we suggest a new solution for mSCTP combing Address Handling Function (AHF) and Simultaneous Mobility Detection Function (SMDF) as well as Name Server (NS), to cope with the problem of simultaneous mobility. Our preliminary results show that the solution has performance as good as the standard mSCTP in a non-simultaneous mobility 
situation in term of packet loss for low rate stream like voice over IP. The drawback is that some modifications are needed to SCTP protocol.

The rest of the paper is organized as follows. In section II, simultaneous mobility and problems caused by it are illustrated. A simultaneous mobility management solution is advanced in section III. Evaluation of the solution is shown in section IV, and we conclude the paper in section V.

\section{Simultaneous Mobility}

Simultaneous mobility refers to the case when both endpoints of a communication session are mobile and they move at about the same time [4]. The movement of endpoint refers to the change of logical location with respect to network access point rather than user geographic position. This means that simultaneous mobility is a logical concept rather than a physical one.

There are four types of mobility [8]: terminal mobility, user mobility, session mobility and service mobility. Terminal mobility is also known as mid-session mobility. In this following, we focus on the terminal mobility only.

It is important to point out that both control message and data may experience the simultaneous mobility problem. In the following, we focus on the control messages only. This is because, compared to payload data, control messages play a more important role in maintaining the communication session. In other words, we consider that, if a suggested solution can tackle the simultaneous mobility problem of control messages, then the probability is high that the suggested solution is able to solve the problem of data messages as well.

The main problem connected with simultaneous mobility is losing the Binding Update (BU) [9] sent by mobile entities Mobile Endpoint (ME)s because it is sent to a previous address of the peer that is moving at around the same time. Both BUs may get lost in this case because of belated arrivals. Other factors, like network congestion, node failure and path error may result in the loss of BUs as well. As a consequence of this, we may have packet losses and longer packet delays. The session is thus suspended and eventually broken, with the consequence of service disruption. The simultaneous mobility problem of mSCTP occurs during the exchanging of IP address binding update using ASCONF and ASCONF-ACK chunks, as shown in Fig. 1. For each ASCONF chunk or ASCONF-ACK chunk, referred to the peer's receiving IP address state, it may arrive before the receiving IP address becomes unreachable (so-called In-Time arrival) or after that (so-called Belated arrival).

\section{Suggested Solution}

Because mSCTP entity does not have a global unique identification during the association life span, it can not resolve the peer's latest IP address after the simultaneous mobility problem occurs. Further-more, mSCTP can not acquire the state of IP address owned by its host, thus another function entity should be employed to trigger the address binding update. In other words, mSCTP is only capable of sending and receiving address binding update. Which IP address should

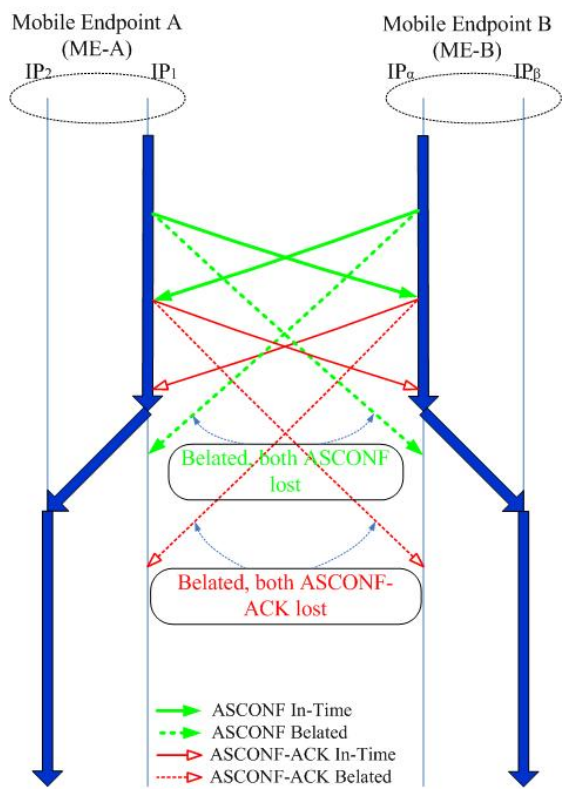

Figure 1. Illustration of simultaneous mobility problem based on ASCONF and ASCONF-ACK arrival time [4]

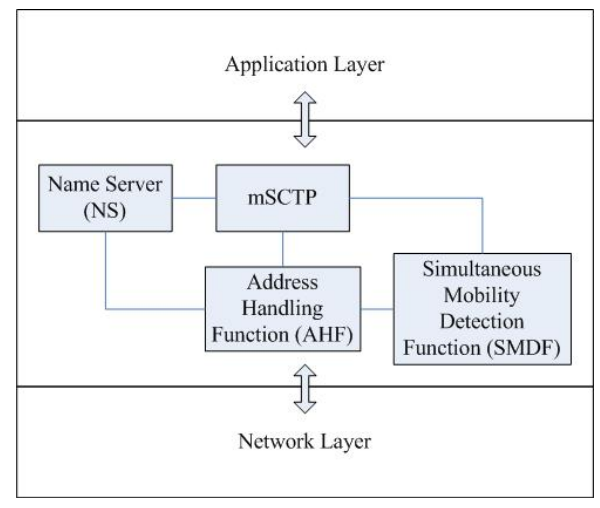

Figure 2. Solution Architecture

be updated and when to send binding update are out of its scope. All in all, the absence of address management of mSCTP prevents it from handling simultaneous mobility problem perfectly.

In order to enhance the mSCTP handling the simultaneous mobility problem, we advance a new solution combining AHF, SMDF and NS, as shown in Fig. 2. AHF is responsible for monitoring the changing of IP address in the mobile endpoint. When the mobile endpoint gets a new IP address through DHCP, PPP or GTP, or it loses its IP address due to the detachment from access point, AHF triggers an event to notify mSCTP function to send an IP address update to its peer and the NS. Meanwhile, SMDF grabs information from the AHF and mSCTP, including state of path, cwnd, number of un-acknowledged chunks and other related statistics of SCTP association. Based on all the information gathered and the time receiving the address changing event from AHF, a decision is made if a simultaneous mobility happens. This decision is sent to the AHF to trigger a query message to NS to get the latest IP address information of its peer. NS is responsible 
for maintaining the binding database and Host Name Address (HNA) resolution. It acts as a pro-active query proxy server for binding updates from endpoints as well. That means NS is not only responsible for the response of name resolution query, but also keeps a copy of the query request for the time $T$. If it gets any updates from the queried address during the particular time, then it actively forwards the update to the sender of query, as shown in Fig. 3 .

Here, we suggest using the HNA parameter in INIT chunk [1], [2] to exchange global unique identifier given that the address type of SCTP includes not only IPv4 and IPv6 addresses but also HNA. HNA is initially designed to work across a NAT box. However, in most cases the HNA is not routable. A NS is therefore introduced to resolve the HNA to get reachable addresses like IPv4, IPv6 or other routable addresses. That means when the simultaneous mobility problem occurs, both endpoints query the NS and acquire the peer's latest reachable address.

Fig. 4 shows the INIT packet structure. The HNA is a variable-length optional field, which may be a host domain name, a dotted-decimal number, a Universal Unique ID (UUID) [10] or a randomly generated string by endpoint itself.

The signaling procedure of this solution is shown in Fig. 5. First of all, both Mobile Endpoint A and B register HNA to the NS (steps 1-2 in Fig. 5). After that, the Mobile Endpoint $\mathrm{A}$ and $\mathrm{B}$ setup an end-to-end association by exchanging control chunks INIT, INIT-ACK, Cookie-Echo and CookieACK similar to the standard SCTP protocol (steps 3-6 in Fig. 5). During the establishment of the SCTP association, endpoints $\mathrm{A}$ and $\mathrm{B}$ exchange HNA by setting the optional parameter of the INIT chunk and the responding INIT-ACK chunk (steps 3 and 4 in Fig. 5). After setting up the association, both endpoints resolve the host name by sending an query to a specified NS by using another SCTP association established between endpoint and the Name Server. The acquired routable IP addresses are then added to the transport address list (steps 7-10 in Fig. 5). Should one or more IP addresses be provided by response, then one of them will be selected as the primary address for the transmitting data and control chunks. The other addresses act as backup or alternative address.

When a ME moves to a new area, it first gets a new IP address, then the AHF detects the changing of IP address, it triggers the mSCTP updating the binding of IP address and HNA to NS and the corresponding endpoint (steps 11-13 in Fig. 5). Because NS is stationary, no BU is late, and the $\mathrm{BU}$ is received by NS. For the binding update to the peer, however, it may be late arrival and gets lost as a consequence of simultaneous mobility. Both mobile endpoints A and B should detect the simultaneous mobility and initiate requests to the NS to get the latest peer address (steps 14-15).

\section{General Evaluation}

\section{A. Setup}

We investigate the performance of the solution during simultaneous mobility situation in terms of packet loss rate and bandwidth in a lab environment. For doing this, we use two Mobile Endpoints, a Name Server, two Link Delay Simulators and a Control Node, as shown in Fig. 6.

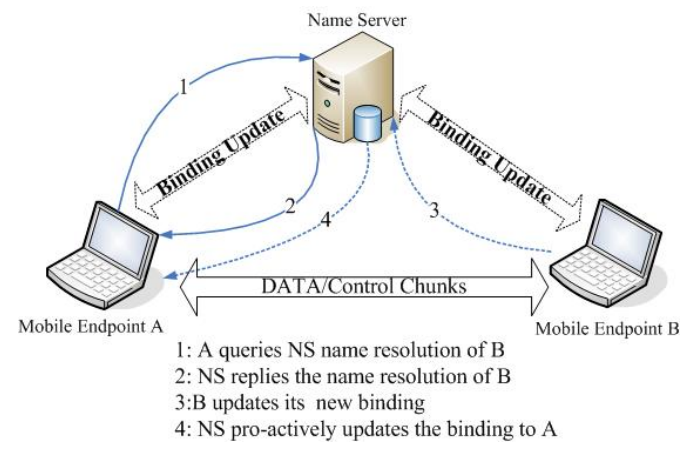

Figure 3. Pro-active Query Response

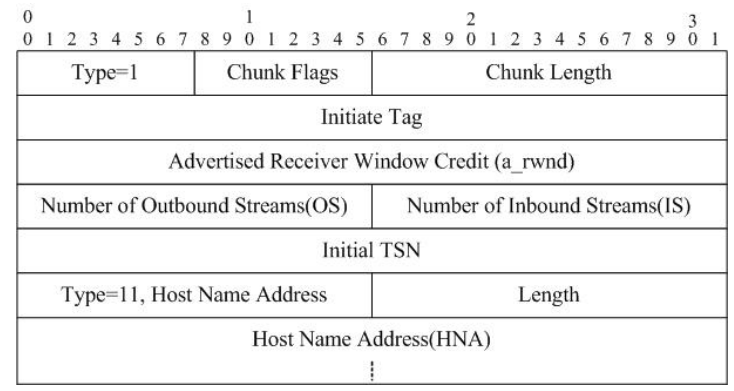

Figure 4. INIT chunk structure

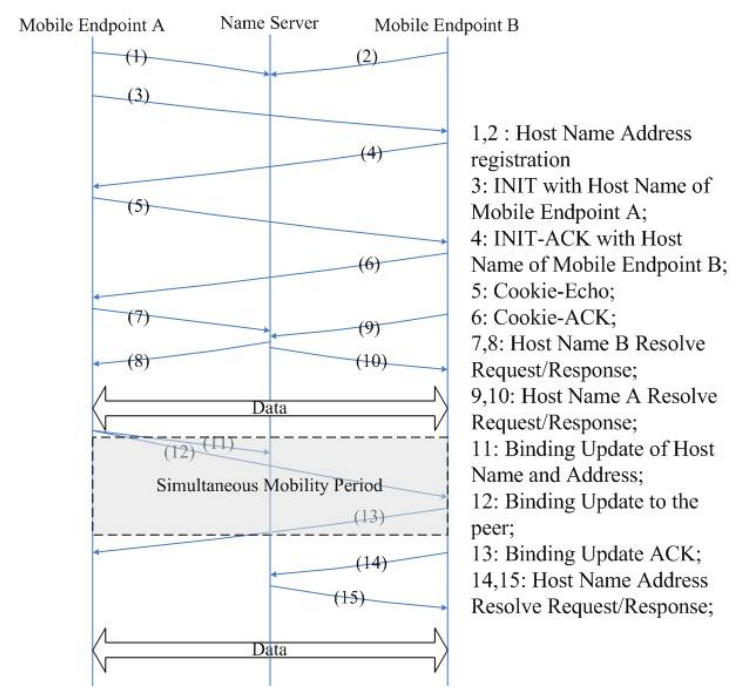

Figure 5. Procedure of exchanging association setup and address resolution

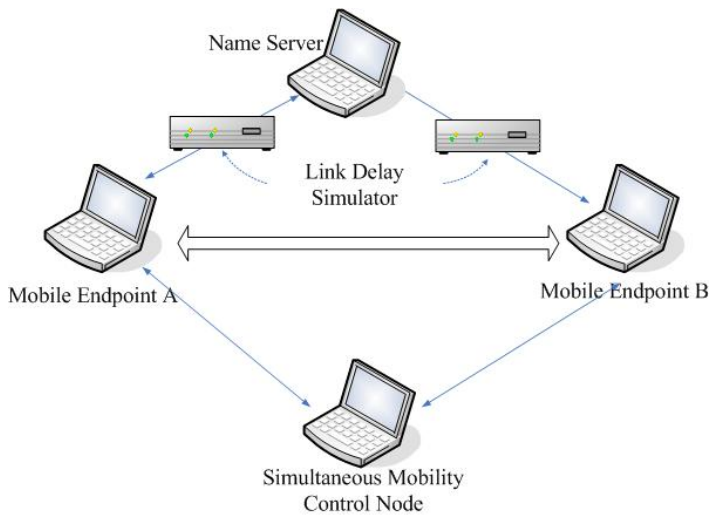

Figure 6. Evaluation Environment 
Table I

Test CAsE DetaiL

\begin{tabular}{|c|c|c|c|}
\hline No. & Test Case & Rate & Reliability \\
\hline \hline 1 & RTVO & $64 \mathrm{kbps}$ & Partial, TTL $=800 \mathrm{~ms}$ \\
\hline 2 & RTVD & $1.8432 \mathrm{Mbps}$ & Partial, TTL $=800 \mathrm{~ms}$ \\
\hline 3 & nRTLO & $64 \mathrm{kbps}$ & Full, TTL $=0 \mathrm{~ms}$ \\
\hline 4 & nRTHI & $1.8432 \mathrm{Mbps}$ & Full, TTL $=0 \mathrm{~ms}$ \\
\hline
\end{tabular}

Two notebooks are used to act as Mobile Endpoints A and B. On the Ethernet physical interface of each notebook, two IP addresses are configured, labeled as $I P_{1}, I P_{2}$ or $I P_{\alpha}, I P_{\beta}$ (see Fig. 1). Actually, the endpoints are not mobile. We use instead the Control Node to change the IP address of interfaces, which is considered to be a similar form as real mobility.

Two Link Delay Simulators are used to introduce latency between the Mobile Endpoint and the Name Server, to simulate the round trip delay and time detecting the simultaneous mobility. Binding update to or from the Name Server is delayed by 100,500 and $1000 \mathrm{~ms}$, respectively. Link Delay Simulator runs on the same host of Mobile Endpoint A and Mobile Endpoint B, respectively.

The Name Server runs on the third notebook together with Control Node. Control Node is responsible to control the status of IP address on endpoints A and B. By sending the control command to both mobile endpoints deleting or adding IP address, the simultaneous mobility is simulated.

Four test cases are considered to simulate Real-Time VOice (RTVO) stream, Real-Time ViDeo (RTVD) stream, non-RealTime LOw (nRTLO) rate traffic and non-Real-Time HIgh (nRTHI) rate traffic, respectively. The details of each stream used in test cases are listed in Table I. We employ partially reliable extension [11] of SCTP to transmit stream in test cases 1 and 2. Partially reliable transport service is defined as a service that allows the user to specify, on a per message basis, the rules governing how persistent the transport service should be in attempting to send the message to the receiver [11]. This service allows the service user to indicate a limit on the duration of time that the sender should try to transmit/retransmit the message. One example of partially reliable service is "timed reliability" service like VoIP, where generating a new packet can provide better voice quality than retransmitting the old one.

For the partial reliability service, the life time of packet is $800 \mathrm{~ms}$, which means that the packet will be discarded if no acknowledgment is received in $800 \mathrm{~ms}$ after being sent.

\section{B. Evaluation Results}

The first set of experiments (Test Case 1 and 3) is performed with Mobile Endpoint B sending a low rate $64 \mathrm{kbps}$ stream to Mobile Endpoint A. The traffic is sent in a fully reliable way and partially reliable way, respectively.

Fig. 7 shows the data rate received by the Mobile Endpoint A during the entire simultaneous mobility period for different time delays and different streams. Real time stream and nonreal time traffic show similar bandwidth utilization during the simultaneous mobility period. The data is buffered in the Mobile Endpoint B when the initial primary path broke due to

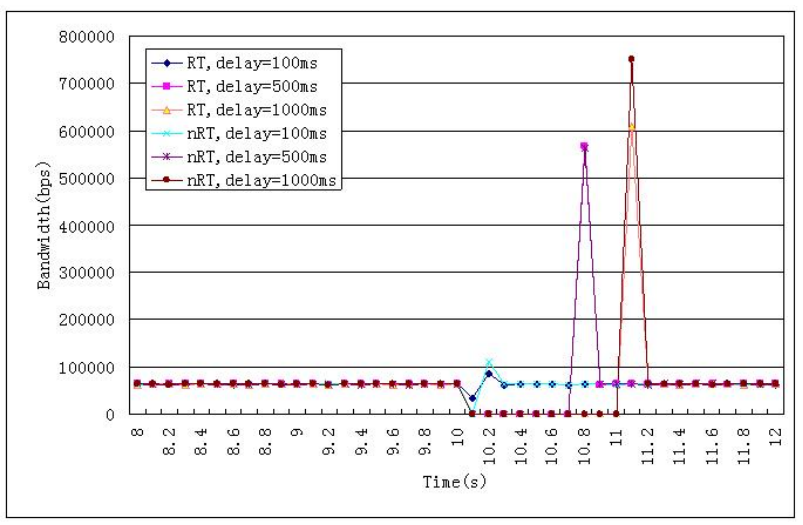

Figure 7. Bandwidth for Low Rate Stream

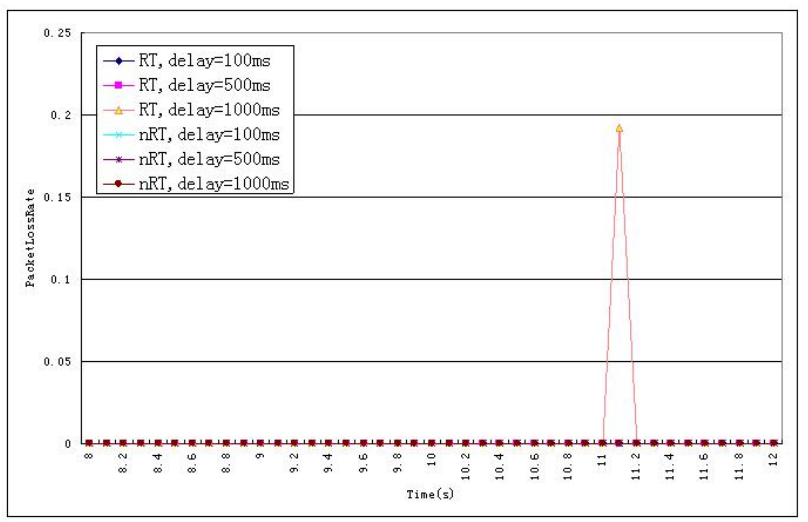

Figure 8. Packet Loss Rate for Low rate Stream

simultaneous mobility, and then it is retransmitted in a burst after the second path is reestablished. Thus the bandwidth used shows a spike after the handover. From Fig. 8, we observe that only the real time stream with $1000 \mathrm{~ms}$ delay suffers from packet loss because the sending mobile endpoint discards the packet given that it does not get any acknowledgment from the designated receiver in $800 \mathrm{~ms}$. Regarding the fully reliable service, no packet loss is observed given that the buffer is big enough to accommodate all the packet in one second less interruption.

The second set of experiments (Test Case 2 and 4) is conducted with Mobile Endpoint B sending a higher rate 1.8432 Mbps stream to Mobile Endpoint A. The traffic is sent in a fully reliable way and partial reliable way, respectively.

Fig. 9 shows the data rate received by the Mobile Endpoint A during the entire simultaneous mobility for different time delays and different streams. Similar to Fig. 7, real time stream and non-real time traffic show similar bandwidth utilization during the simultaneous mobility period. The bandwidth used shows a spike after the handover as well. This is because the data is buffered at the sender side when the initial primary path broke and then retransmitted in a burst after the second path is re-established. In Fig. 10, we observe that not only the real time stream with $500 \mathrm{~ms}$ and $1000 \mathrm{~ms}$ delay but also nonreal time traffic with $500 \mathrm{~ms}$ and $1000 \mathrm{~ms}$ delay suffer from packet loss, and packet loss of real time stream is relatively higher than that of non-real time stream. Compared to the low 


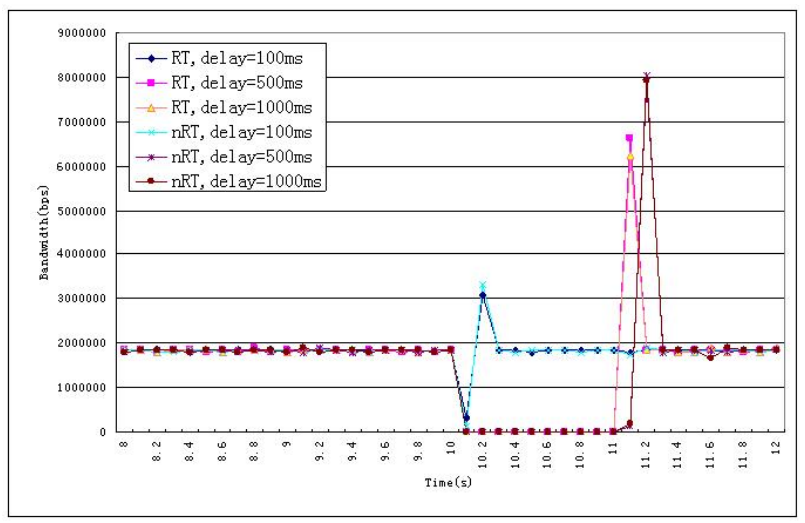

Figure 9. Bandwidth for High Rate Stream

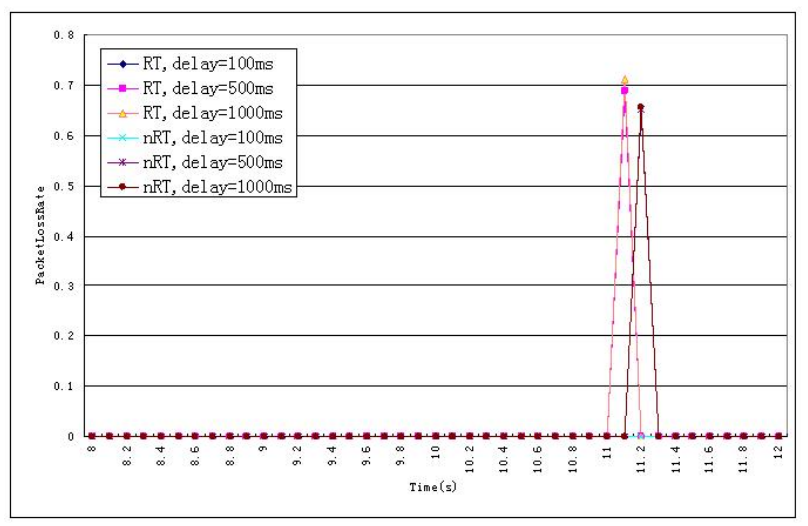

Figure 10. Packet Loss Rate for High Rate Stream

rate stream with same delay in Fig. 8, high rate stream suffers much higher packet loss rate. In Fig. 8 and Fig. 10, both stream and traffic with $100 \mathrm{~ms}$ delay do not suffer from packet loss, similar to standard mSCTP handover between two interfaces. That means buffer size at the sender side plays an important role in reducing packet loss of high rate stream like video and well dimensioned buffer can reduce the packet loss rate and improve the performance during handover.

For bandwidth results shown in Fig. 7 and Fig. 9, they display similar form as those in [5]. In Fig. 7 and Fig. 9, we observe the packet rate becomes normal shortly after regaining the new connection. The fact proves that the solution works effectively to recover the association from the simultaneous mobility problem.

\section{CONCLUSIONS}

In this paper, a solution based on Host Name Address (HNA) together with additional Name Server (NS) is suggested to solve this problem. Other two functions Address Handling Function (AHF) and Simultaneous Mobility Detection Function (SMDF) are employed to detect the IP address changing and simultaneous mobility. The results of evaluation show that no packet loss were observed by a stream at a relatively low rate like Voice over IP (VoIP). For high rate service, if the association broken time is limited to a scale of $100 \mathrm{~ms}$, we can also get very good performance with the help of buffer. For longer broken time caused by simultaneous mobility, the packet loss is inevitable. Anyhow, the normal transmission is quickly resumed after the network connectivity is regained.

An important advantage of the suggested solution is that it is transparent to upper layer applications. It is mSCTP that handles the simultaneous mobility problem, and an upper layer application can not even sense the problem. Another important advantage is that no change is needed to be done at the network layer. Further, a reliable connection using SCTP is established for address updating instead of a connectionless UDP session used in Dynamic DNS (DDNS) solution.

However, the suggested solution has several drawbacks as well. The first is that we need to modify the SCTP protocol. Furthermore, we need one more SCTP association for transmitting HNA binding update and name query as well as response. Reusing the SCTP association for name resolution between different applications can be a solution to reduce the number of SCTP associations in an endpoint.

Future research is about deploying the NS system in a largescale network. Another important issue is how to find the nearest NS with reference to minimizing the name resolution delay, which is critical to the performance of simultaneous mobility and seamless roaming. Finally, another direction is about extending the function of the NS in the sense that more information is stored in the NS like, e.g., geographic location and point of attachment to predict the mobility and minimize the simultaneous mobility detection time.

\section{REFERENCES}

[1] R. Stewart, Ed., "Stream Control Transmission Protocol", IETF RFC 4960, September 2007.

[2] R. Stewart, Q. Xie, K.Morneault, C. Sharp, H. Schwarzbauer, T. Taylor, I. Rytina, M. Kalla, L. Zhang, V. Paxson, "Stream Control Transmission Protocol", IETF RFC 2960, October 2000.

[3] R. Stewart, Q. Xie, M. Tuexen, S. Maruyama, M. Kozuka, "Stream Control Transmission Protocol (SCTP) Dynamic Address Reconfiguration", IETF RFC 5061, September 2007.

[4] K. Daniel Wong, A. Dutta, H. Schulzrinne, K. Young, "Simultaneous Mobility: Analytical Framework, Theorems and Solutions", WIRELESS COMMUNICATIONS AND MOBILE COMPUTING. Wirel. Commun. Mob. Comput. 2007, 7:623-642.

[5] Thomas Dreibholz, Andreas Jungmaier, Michael Tuxen, "A New Scheme for IP-based Internet Mobility", The 28th Annual IEEE Conference on Local Computer Networks, Germany, October 2003.

[6] Seok J. Koh, Mee Jeong Lee, Maximilian Riegel, Mary Li Ma, Michael Tüexen, "Mobile SCTP for Transport Layer Mobility", IETF draft-sjkohsctp-mobility-04.txt, June 2004.

[7] P. Vixie, Ed., S. Thomson, Y. Rekhtergoal, J. Bound, "Dynamic Updates in the Domain Name System (DNS UPDATE)", IETF RFC 2136, April 1997.

[8] Popescu A.,'D5.1 - Conceptual Architecture for Seamless Roaming", deliverable D5.1, Mobicome, June 2008.

[9] J. Manner, Ed.,M. Kojo, Ed., "Mobility Related Terminology", IETF RFC 3753, June 2004.

[10] P. Leach, M. Mealling, R. Salz,’A Universally Unique IDentifier (UUID) URN Namespace", IETF RFC 4122, July 2005.

[11] R. Stewart, M. Ramalho, Q. Xie, P. Conrad, "Stream Control Transmission Protocol (SCTP) Partial Reliability Extension", IETF RFC 3758, May 2004. 